\title{
BMJ Open Effects of traditional qigong exercise on ankylosing spondylitis: a protocol for systematic reviews and meta-analysis
}

\author{
Wei Liu (D) , ${ }^{1,2}$ Yihua Fan (D) , ${ }^{1,2}$ Renhong Wan (D) , ${ }^{3}$ Longmei Zhao, ${ }^{1}$ Hang Lu, ${ }^{1}$ \\ Rongjun Liao, ${ }^{1}$ Zhining Zhuang, ${ }^{1}$ Xiaoping Guo ${ }^{1}$
}

To cite: Liu W, Fan Y, Wan R, et al. Effects of traditional qigong exercise on ankylosing spondylitis: a protocol for systematic reviews and meta-analysis. BMJ Open 2021;11:e046188. doi:10.1136/ bmjopen-2020-046188

- Prepublication history and additional supplemental material for this paper are available online. To view these files, please visit the journal online (http://dx.doi.org/10.1136/ bmjopen-2020-046188)

WL and YF contributed equally.

WL and YF are joint first authors.

Received 25 October 2020

Revised 27 March 2021

Accepted 09 April 2021

Check for updates

(C) Author(s) (or their employer(s)) 2021. Re-use permitted under CC BY-NC. No commercial re-use. See rights and permissions. Published by BMJ.

${ }^{1}$ Department of Rheumatism and Immunology, First Teaching Hospital of Tianjin University of Traditional Chinese Medicine,

Tianjin, China

${ }^{2}$ National Clinical Research Center for Chinese Medicine Acupuncture and Moxibustion, Tianjin, China

${ }^{3}$ Graduate School, Tianjin University of Traditional Chinese Medicine, Tianjin, China

Correspondence to

Dr Wei Liu;

fengshiliuwei@163.com

\section{ABSTRACT}

Introduction Ankylosing spondylitis (AS) is a recurrent autoimmune disease that is associated with a high disability rate, which has a profound negative impact on daily life. It has been clinically reported that traditional qigong exercise has certain therapeutic advantages in treating AS; however, this postulation is not evidencebased. Therefore, this study will aim at systematically appraising the validity and safety of traditional qigong exercise in treating AS.

Methods and analysis We searched the English (PubMed, Embase, Web of Science and the Cochrane Library) and Chinese databases (China National Knowledge Infrastructure, Wan fang, VIP and Chinese biomedical database) for relevant information. Additionally, the Baidu Academic and Google Academic databases were manually searched to mine relevant information. Randomised controlled clinical trials of traditional qigong exercise in treating AS were searched from the establishment of the database to September 2020. Quality articles were extracted and independently evaluated by two researchers before inclusion in this study. Meta-analysis of the included articles was performed using the RevMan V.5.3 software.

Ethics and dissemination This study will not involve primary data collection, and formal ethics approval will, therefore, not be required. We aim to publish this systematic review in a peer-reviewed journal. Trial registration number CRD42020207057.

\section{INTRODUCTION}

Ankylosing spondylitis (AS) is a chronic systemic autoimmune disease that affects the sacroiliac joints, axial skeleton, peripheral joints, tendon-ligament attachment points and other bony organisations. ${ }^{12}$ It is often accompanied by finger inflammation (25\%$50 \%)$, uveitis $(25 \%-40 \%)$, inflammatory bowel disease $(26 \%)$ and psoriasis $(10 \%)$, all of which affect the patients' quality of life. ${ }^{3}$

The average prevalence of AS per 10000 is 23.8 in Europe, 16.7 in Asia, 31.9 in North America, 10.2 in Latin America and 7.4 in Africa. ${ }^{4}$ This disease is prevalent among those aged between 20 and 30 years and affects more males than females. At its onset, AS is asymptomatic, thus its diagnosis is delayed by

\section{Strengths and limitations of this study}

This study will present, for the first time, a comprehensive systematic evaluation and meta-analysis of traditional functional therapy in treating ankylosing spondylitis (AS).

We will offer an evidence-based framework for using traditional methods to treat AS in a clinical setting.

- Due to the diverse range of functional exercises, heterogeneity risk may increase.

- We only included studies in Chinese and English languages, which may increase the risk of bias.

5-6years. ${ }^{23}$ It presents with various clinical features in its clinical course, with a third of the patients developing severe disabilities. Back pain, ankylosis, limited chest expansion and limited spinal activity often occur in the early stages of the disease, resulting in occupational disability and economic burden. ${ }^{5}$ Currently, non-steroidal anti-inflammatory agents are the main therapeutic options for symptomatic AS. However, these medications exert various side effects to the cardiovascular system, gastrointestinal tract and kidney. Besides, the anti-tumour necrosis factor (TNF) biologics used to treat AS are associated with adverse side effects and are expensive. ${ }^{6}$ Exercise is the main scheme for non-drug treatment of AS. Appropriate exercise relieves pain and relaxes joints, which improves health and life quality. ${ }^{6}$ Reasonable exercise programmes can promote the recovery of body functions in AS. ${ }^{7}$

Traditional qigong exercises, including qigong, Tai Chi, Baduanjin, represent one of the preventive and healthcare methods with the characteristics of traditional Chinese medicine, which have been widely used to treat Parkinson's disease, non-specific low back pain, cancer and other conditions. ${ }^{8-10}$ Its curative effect has been proven to play a complementary and alternative role ${ }^{11}$ in some bone and joint diseases, among other 
diseases. Moreover, qigong exercise has potential values in the rehabilitation of patients with COVID-19. ${ }^{12}$ This exercise can increase muscle flexibility, improve cardiopulmonary functions, enhance joint flexibility, increase muscle strength and relieve joint pain, which are beneficial for AS. ${ }^{13}$

Traditional qigong exercises, including Tai Chi, Wu Qin Xi, Liu Zi Jue and Yi Jin Jing, among others have been developed in China for more than 1000 years. These exercises aim at integrating breathing exercises into body movements, ${ }^{14}$ mental activities, limb movements and self-massage to promote blood circulation, regulate the immune system, reduce interleukin 6 levels and regulate the inflammatory process. ${ }^{15} 16$ Clinical studies have confirmed that traditional qigong exercises can improve the body's balance and flexibility, improve life quality, as well as alleviate depression and anxiety. ${ }^{17-19}$ Regular exercise enhances immunity by improving $\mathrm{T}$ cell subset distribution in human peripheral blood. ${ }^{20}$ Baduanjin could fully relax muscles, tendons and ligaments in the whole body and to effectively relieve muscle spasm, and to relieve pain. ${ }^{21}$ It improves morning stiffness of the waist and spine in patients with $\mathrm{AS}^{21}$ and relieves body fatigue. ${ }^{22}$ Tai Chi can promote patients' flexibility, improve the function of cardiovascular system, enhance muscle strength, increase joint flexibility, reduce joint pain and facilitate patient with AS recovery. ${ }^{102}$ Mawangdui guidance can influence the structure and function of anterior insula and anterior cingulate cortex to regulate emotions, ${ }^{15}$ improve mood, improve physical flexibility and relieve pain in patients with AS. ${ }^{24}$

Randomised controlled trials (RCTs) have confirmed the effectiveness ${ }^{22} 2526$ of traditional qigong exercises in treating AS. However, discrepancies among study designs and curative effects reported in different clinical trials have inhibited establishment of its curative effect. Therefore, this systematic review and meta-analysis will aim at evaluating the efficacy and safety of traditional qigong exercise in the treatment of AS and to provide evidencebased information for clinical applications.

\section{METHODS}

\section{Study registration}

The protocol for this study was drafted under the systematic review and meta-analysis preferred reporting project (PRISMA-P). The protocol and registration information are available at https://www.crd.york.ac.uk/prospero/display_record.php?RecordID $=207057$. This study commenced on 23 August 2020 and is planned to be completed before 31 July 2021.

\section{Inclusion criteria}

Studies

RCT studies, in Chinese and English languages, on the treatment of AS using traditional qigong exercise.

\section{Participants}

Patients with a definite diagnosis of AS (according to the modified New York criteria for $\mathrm{AS}^{26}$ ), regardless of nationality, race, age, gender and disease duration among others.

\section{Types of interventions}

Treatment groups were subjected to traditional qigong exercise (including Tai Chi, Baduanjin, Shi'erduanjin, changing tendon exercise, five mimic-animal exercises, sixcharacter formula, five elements balance work, Longmen five elements skill, gymnastic qigong, Mawangdui guiding technique and qigong) combined with western medicine. There were no restrictions regarding the type of traditional qigong exercise, the type of western medicine, treatment frequency and treatment course, among other factors. The control group was only administered with the drug used in the treatment group.

\section{Outcome measures}

Primary outcomes:

1. Overall efficiency (we refer to the clinical efficacy evaluation of AS in guiding principles for clinical research of new Chinese medicine. ${ }^{27}$ Clinical cure: $\geq 75 \%$ overall improvement in the main symptoms and signs, with normal or significantly improved or close to normal erythrocyte sedimentation rate (ESR) and $\mathrm{C}$ reactive protein (CPR); significant effect: $\geq 50 \%$ overall improvement in main symptoms and signs with improved ESR and CPR; effective: $\geq 30 \%$ overall improvement in major symptoms and signs, with improved or non-improved ESR and CPR; inefficacy: $<30 \%$ overall improvement in main symptoms and signs with unimproved ESR and CPR. Overall efficiency was defined as clinical cure+significant effect+effective/total number $\times 100 \%$ ).

2. Bath Ankylosing Spondylitis Disease Activity Index. ${ }^{28}$ Secondary outcomes:

1. Functional ability (measured using the Bath Ankylosing Spondylitis Functional Index. ${ }^{29}$

2. Ankylosing Spondylitis Metrology Index. ${ }^{29}$

3. Chest expansion $(\mathrm{cm})$.

4. Nocturnal spinal pain.

5. Laboratory indicators, including erythrocyte sedimentation rate and $\mathrm{C}$ reactive protein response.

6 . Incidences of adverse reactions.

\section{Exclusion criteria}

1. Republished papers.

2. Articles published as abstracts or with incomplete data, or complete articles could not be obtained even after contacting the author(s).

3. Studies exhibiting a high-risk bias. ${ }^{30}$

4. Studies whose treatments were combined with other traditional Chinese medicine methods, such as acupuncture, moxibustion and Chinese herbal medicines.

5. Studies whose patients were involved in other sports (such as swimming, yoga, etc).

6. Studies without the relevant outcome indicators.

\section{Search terms for retrieval of studies}

'Traditional Kong Fu', 'Qigong', 'Baduan Jin', 'Tai Chi', 'Ankylosing Spondylitis' and 'Ankylosing Spondylitis' were 
used as Chinese search words in the Chinese databases, which included the China National Knowledge Infrastructure, Wan fang, Data Knowledge Service Platform and VIP Chinese periodical service platform (VIP). We used 'Traditional qigong exercise', 'Wuqinxi', 'Bechterew Disease', 'Ankylosing spondylitis' as English search terms in the English databases, including PubMed, EMBASE, Web of Science and the Cochrane Library, and to manually retrieve relevant documents on the Baidu Academic and Google Academic databases. Retrieval time was from establishment of the databases to September 2020. All local and foreign documents on AS treatment with traditional methods were collected. The data retrieval strategy from the PubMed database was as shown in online supplemental table 1 .

\section{Data collection and analysis}

\section{Data extraction and management}

We referred to the research selection method in the Cochrane collaboration Network system Evaluator Manual V.5.0. Based on the PRISMA flowchart, two researchers will use the EndNote X9 reference management software to independently screen, cross-check and verify the retrieved documents according to the study's inclusion and exclusion criteria. In case of divergent opinions, the researchers will negotiate with a third researcher to achieve a consensus. Meanwhile, we will use Excel 2013 software to extract relevant information, which included:

1. Clinical research (the title of the study, first author name, year and month of publication, sample size, sex ratios, average age and average disease course).

2. Intervention measures in the treatment and control groups (types, frequency, and duration of treatment by traditional qigong; types, dosage forms, frequency, and duration in the treatment and control groups).

3. Risk bias assessment factors in RCT.

4. Required outcome indicators.

The process of screening the selected studies was as shown in figure 1.

\section{Assessment of the risk of bias}

Risk bias for the included studies will be evaluated using a built-in risk bias assessment tool in Review Manager (RevMan) V.5.3 software (the Cochrane collaboration's tool for assessing the risk of bias). Accordingly, two researchers will determine whether the selected studies had a low-risk, unclear or a high-risk bias based on the six aspects of adequate sequence gene ratio, allocation concealment, blinding, incomplete outcome data, selective reporting, other bias, and cross-checked each of them. In case there will be differences, they will discuss or involve third party researchers to arrive at a consensus.

\section{Measures of treatment effect}

The RevMan V.5.3 and STATA software packages were used to synthesise all the obtained data. Dichotomy data results were presented as risk ratios and weighted average differences at a $95 \%$ CI. On the other hand, continuous data were standardised and presented as average differences at a $95 \%$ confidence level.

\section{Methods for dealing with missing data}

When the required data are incomplete or not reported in a study, the reviewer will contact the corresponding author or other authors by telephone or email to obtain the missing/incomplete data. We will use descriptive analysis instead of a meta-analysis.

\section{Unit of analysis issues}

Before statistical analyses, the units of different search results will be converted into the International System of Units.

\section{Data synthesis}

We will use the RevMan V.5.3 software for meta-analysis. Binary variables will be expressed as degrees of relative risk (relative ratio). For continuous outcomes, we will use the Weighted Mean Difference to determine the consistency of the measurement tool and unit of measurement at a $95 \%$ CI. The magnitude of the effect will be expressed as the Standard Mean Difference at 95\% CI. Heterogeneity among studies will be determined by the $\chi^{2}$ and $\mathrm{I}^{2}$ values. Meta-analysis will be performed using a fixed-effects model. Heterogeneity will be deemed low if $\mathrm{p} \geq 0.1$ and $\mathrm{I}^{2} \leq 50 \%$ and high if $\mathrm{p}<0.1$ and personality $\mathrm{I}^{2}$ $>50 \%$. The heterogeneity source will be analysed whenever detected and deemed high. Besides, clinical data heterogeneity will be subjected to subgroup analyses, and statistical heterogeneity analysis will be considered in case of unclear clinical or methodological heterogeneity. When appropriate, the random-effect model will be used for statistical heterogeneity analysis.

\section{Subgroup analysis and investigation of heterogeneity}

In case of significant clinical heterogeneity between studies $\left(\mathrm{I}^{2} \geq 50 \%\right)$, subgroup analyses will be performed to explore the source(s) of heterogeneity.

For example, subgroup analysis will be performed based on types of functional methods, the course of treatment, and the different drug treatment schemes in the control group.

\section{Sensitivity analysis}

Sensitivity analysis will be performed based on the Cochrane manual's recommendations to test the stability of the indexed meta-analysis results by the elimination method.

\section{Assessment of the publication bias}

A funnel chart will be used to assess publication bias in outcome measures from more than 10 studies. Additionally, the Egger's and Begg's tests will be used to determine potential publication bias.

\section{Evaluation of evidence quality}

Based on bias risk, consistency, directness, precision and publication bias, the Grading of Recommendations 


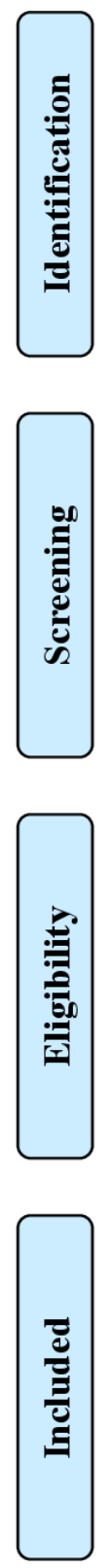

Records identified through database searching

$$
(\mathrm{n}=)
$$

Additional records identified through other sources

$$
(\mathrm{n}=)
$$

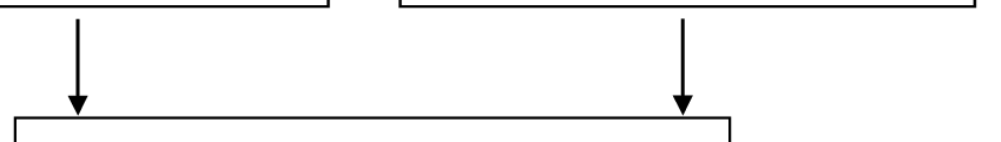

Records after duplicates removed $(\mathrm{n}=)$

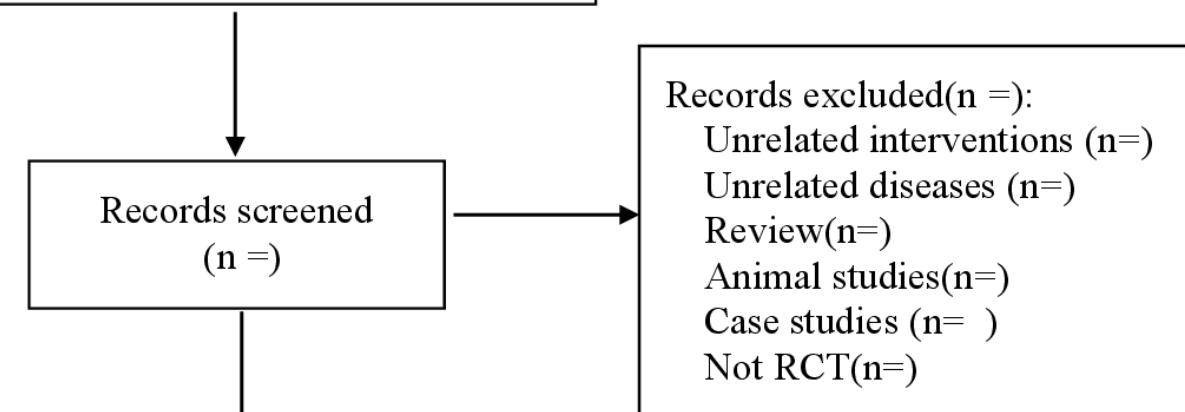

Full-text articles assessed for eligibility $(\mathrm{n}=)$

Full-text articles excluded $(\mathrm{n}=)$ :

Unrelated interventions $(\mathrm{n}=)$ Inconsistent outcome $(\mathrm{n}=)$ Incomplete data $(\mathrm{n}=)$ Repeat published( $\mathrm{n}=)$ Not RCT $(\mathrm{n}=)$

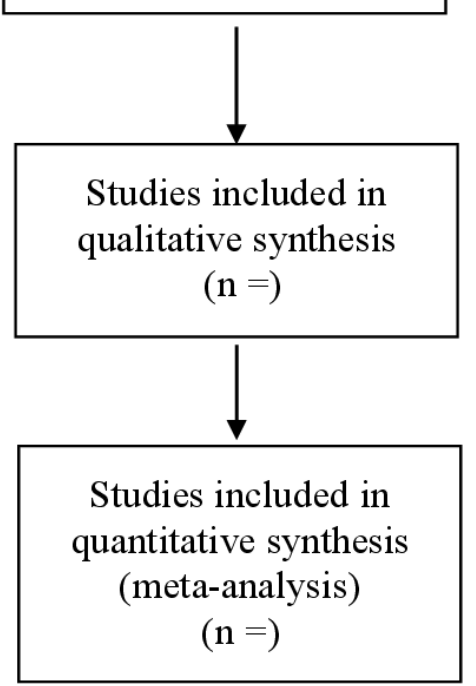

Figure 1 Flow diagram.

Assessment, Development and Evaluation will be used to assess the quality of evidence in this study. The quality of evidence will be rated as high, moderate, low and very low, respectively.

\section{Patient and public involvement}

Patients and the public did not participate directly in this review study as we only used secondary data provided in the literature and other sources.

\section{DISCUSSION}

The aetiology of AS has not been fully elucidated. However, it has been shown that it is closely associated with genetic and environmental factors. ${ }^{2}{ }^{31}$ The current therapeutic options for AS involve pain alleviation, recovery of physical functions related to daily life and occupational activities, as well as delaying structural damages responsible for physical impairments. ${ }^{6}$ However, these treatment strategies are not effective or universally affordable. 
Additionally, affected patients endure physical pain as well as psychological pressures such as depression. ${ }^{32}$ Therefore, it is important to develop new supplements and alternatives to treat AS.

Based on the Assessment of SpondyloArthrities guidelines, patients with AS should exercise regularly. ${ }^{33}$ Clinical studies have confirmed that exercises such as Tai Chi, Pilates and swimming can alleviate pain, improve spinal activity and quality of life in patients with AS. ${ }^{34}{ }^{35}$ Traditional exercise methods, which originated from exercise methods in ancient China, have formed a complete sports system and have been internationally recognised. At home and abroad, traditional exercises have been used to treat many diseases. Traditional exercises can assist in improving symptoms and motion in patients with AS, which is of great significance to their prognosis.

However, data from RCTs are not consistent. With the increasing number of clinical trials, it is imperative to evaluate the efficacy of traditional exercises in systematically treating AS. In this study, we have summarised the latest evidence on the efficacy of traditional qigong exercise in AS treatment. Pain and limited movements of the spinal and hip joints are the main clinical manifestations of this disease, therefore, we focused on the patient's spinal function score. Laboratory indicators are important markers for establishing whether a disease is in the active phase. Therefore, through clinical symptom scores and laboratory indicators, this meta-analysis will provide a convincing conclusion regarding the effectiveness of traditional exercises for AS. This study will be beneficial to both patients and decision-makers in clinical practice. Clinical applications of traditional exercises can improve the quality of life for patients with AS, thereby saving medical costs.

It should be noted that there might be limitations in this review. There may be some clinical heterogeneities due to differences in the frequency, intensity and course of treatment, as well as the degree of patients' illness among the included studies. In addition, there are differences in the basic treatments of patients, which may have a certain impact on the results of the meta-analysis. Due to language barriers, we only included studies in English and Chinese languages and ignored studies presented in other languages.

\section{Ethics and dissemination}

Approval from an ethics committee will not be required as this systematic review will use publicly available data without directly involving human participants. Our findings will be presented at relevant scientific conferences or disseminated through publication in a peer-reviewed journal.

Acknowledgements WL and YF have contributed equally to this work and are co-first authors. The authors would like to thank Freescience (www.home-forresearchers.com) for the help with the English language.

Contributors This study was conceived by WL, YF and RW. YF and RW drafted the manuscript. LZ, XG, ZZ and YF participated in the design of the data synthesis and analysis scheme. WL, $\mathrm{HL}$ and $\mathrm{RL}$ reviewed and revised the manuscript. All authors have read and approved the publication of the protocol.

Funding This study was financially supported by the National Natural Science Foundation of China (No. 82074377), the National Key Research and Development Project (No. 2018YFC1705201), the Tianjin Science and Technology Commission Program (No. 2019ZD12), Tianjin Key Specialty Program (20200602-1) and the Special Scientific Research for Traditional Chinese Medicine (No. 201507001-07).

Competing interests None declared.

Patient consent for publication Not required.

Provenance and peer review Not commissioned; externally peer reviewed.

Supplemental material This content has been supplied by the author(s). It has not been vetted by BMJ Publishing Group Limited (BMJ) and may not have been peer-reviewed. Any opinions or recommendations discussed are solely those of the author(s) and are not endorsed by BMJ. BMJ disclaims all liability and responsibility arising from any reliance placed on the content. Where the content includes any translated material, BMJ does not warrant the accuracy and reliability of the translations (including but not limited to local regulations, clinical guidelines, terminology, drug names and drug dosages), and is not responsible for any error and/or omissions arising from translation and adaptation or otherwise.

Open access This is an open access article distributed in accordance with the Creative Commons Attribution Non Commercial (CC BY-NC 4.0) license, which permits others to distribute, remix, adapt, build upon this work non-commercially, and license their derivative works on different terms, provided the original work is properly cited, appropriate credit is given, any changes made indicated, and the use is non-commercial. See: http://creativecommons.org/licenses/by-nc/4.0/.

\section{ORCID iDs}

Wei Liu http://orcid.org/0000-0002-3364-0966

Yihua Fan http://orcid.org/0000-0003-4730-4004

Renhong Wan http://orcid.org/0000-0002-1315-1997

\section{REFERENCES}

1 Garcia-Montoya L, Gul H, Emery P. Recent advances in ankylosing spondylitis: understanding the disease and management. F1000Res 2018;7. doi:10.12688/f1000research.14956.1. [Epub ahead of print: 2109 2018].

2 Smith JA. Update on ankylosing spondylitis: current concepts in pathogenesis. Curr Allergy Asthma Rep 2015;15:489.

3 Maxwell LJ, Zochling J, Boonen A, et al. Tnf-Alpha inhibitors for ankylosing spondylitis. Cochrane Database Syst Rev 2015;53:Cd005468.

4 Dean LE, Jones GT, MacDonald AG, et al. Global prevalence of ankylosing spondylitis. Rheumatology 2014;53:650-7.

5 Zochling J, van der Heijde D, Burgos-Vargas R, et al. ASAS/EULAR recommendations for the management of ankylosing spondylitis. Ann Rheum Dis 2006;65:442-52.

6 Moon K-H, Kim Y-T. Medical treatment of ankylosing spondylitis. Hip Pelvis 2014;26:129-35.

7 Pécourneau V, Degboé Y, Barnetche T, et al. Effectiveness of exercise programs in ankylosing spondylitis: a meta-analysis of randomized controlled trials. Arch Phys Med Rehabil 2018;99:383-9.

8 Phattharasupharerk S, Purepong N, Eksakulkla S, et al. Effects of Qigong practice in office workers with chronic non-specific low back pain: a randomized control trial. J Bodyw Mov Ther 2019;23:375-81.

9 Song R, Grabowska W, Park M, et al. The impact of tai chi and Qigong Mind-body exercises on motor and non-motor function and quality of life in Parkinson's disease: a systematic review and metaanalysis. Parkinsonism Relat Disord 2017;41:3-13.

10 Wayne PM, Lee MS, Novakowski J, et al. Tai chi and Qigong for cancer-related symptoms and quality of life: a systematic review and meta-analysis. J Cancer Surviv 2018;12:256-67.

11 Klein PJ, Baumgarden J, Schneider R. Qigong and tai chi as therapeutic exercise: survey of systematic reviews and metaanalyses addressing physical health conditions. Altern Ther Health Med 2019;25:48-53.

12 Feng F, Tuchman S, Denninger JW, et al. Qigong for the prevention, treatment, and rehabilitation of COVID-19 infection in older adults. Am J Geriatr Psychiatry 2020;28:812-9.

13 Chong Z, Yue W. Study on the application of exercise therapy in the treatment of ankylosing spondylitis. $J$ introduction of traditional Chinese Medicine 2015;12:91-3. 
14 Lan C, Chen S-Y, Lai J-S, et al. Tai chi chuan in medicine and health promotion. Evid Based Complement Alternat Med 2013;2013:1-17.

15 Chen X, Cui J, Li R, et al. Dao Yin (a.k.a. Qigong): origin, development, potential mechanisms, and clinical applications. Evid Based Complement Alternat Med 2019;2019:1-11.

16 Matos LC, Sousa CM, Gonçalves M, et al. Qigong as a traditional vegetative biofeedback therapy: long-term conditioning of physiological Mind-body effects. Biomed Res Int 2015;2015:1-6.

17 So WWY, Cai S, Yau SY, et al. The neurophysiological and psychological mechanisms of Qigong as a treatment for depression: a systematic review and meta-analysis. Front Psychiatry 2019;10:820.

18 Wang C-W, Chan CHY, Ho RTH, et al. Managing stress and anxiety through qigong exercise in healthy adults: a systematic review and meta-analysis of randomized controlled trials. BMC Complement Altern Med 2014;14:8

19 Zou L, SasaKi JE, Wang H, et al. A systematic review and metaanalysis Baduanjin Qigong for health benefits: randomized controlled trials. Evid Based Complement Alternat Med 2017;2017:1-17.

20 Jingmei W, Dinghai Y. Effects of "Fitness Qigong Wuqinxi" exercise on T Lymphocyte subsets in Peripheral Blood of the Middle-aged and elderly. J Journal of Beijing Sport University 2006;29:1074-5.

21 Jingti J, Weihong Z, Ying S. Mechanism of Baduanjin in improving clinical symptoms of ankylosing spondylitis a brief discussion on. $J$ Massage and Rehabilitation Medicine 2016;7:3-4.

22 Xie Y, Guo F, Lu Y, et al. A 12-week Baduanjin Qigong exercise improves symptoms of ankylosing spondylitis: a randomized controlled trial. Complement Ther Clin Pract 2019;36:113-9.

23 Yuke S, Huiru W. Exploration on the teaching of fitness Qigong Mawangdui guidance- a clinical follow-up study of ankylosing spondylitis. J fighting 2015;12:86-8.

24 Lee E-N, Kim Y-H, Chung WT, et al. Tai chi for disease activity and flexibility in patients with ankylosing spondylitis--a controlled clinica trial. Evid Based Complement Alternat Med 2008;5:457-62.

25 Deyi C, Mingshan Q, Jinchun C. Observation on the intervention effect of Taiji white Crane brightening wings on ankylosing spondylitis. J Asia-Pacific traditional Medicine 2015;11:70-3.
26 van der Linden S, Valkenburg HA, Cats A. Evaluation of diagnostic criteria for ankylosing spondylitis. A proposal for modification of the new York criteria. Arthritis Rheum 1984;27:361-8.

27 Zheng XY. Guiding principles for clinical research of new Chinese medicine (trial. Beijing: China Medical Science and Technology Press, 2002: 132-4.

28 Zochling J. Measures of symptoms and disease status in ankylosing spondylitis: ankylosing spondylitis disease activity score (ASDAS), ankylosing spondylitis quality of life scale (ASQoL), Bath ankylosing spondylitis disease activity index (BASDAI), Bath ankylosing spondylitis functional index (BASFI), Bath ankylosing spondylitis global score (BAS-G), Bath ankylosing spondylitis Metrology index (BASMI), Dougados functional index (DFI), and health assessment questionnaire for the Spondylarthropathies (HAQ-S). Arthritis Care Res 2011;63 Suppl 11:S47-58.

29 Sieper J, Rudwaleit M, Baraliakos X, et al. The assessment of spondyloarthritis International Society (ASAS) Handbook: a guide to assess spondyloarthritis. Ann Rheum Dis 2009;68 Suppl 2:ii1-44.

30 Zhao J-G, Zeng X-T, Wang J, et al. Association between calcium or vitamin $\mathrm{D}$ supplementation and fracture incidence in communitydwelling older adults: a systematic review and meta-analysis. JAMA 2017;318:2466-82.

31 Hanson A, Brown MA. Genetics and the causes of ankylosing spondylitis. Rheum Dis Clin North Am 2017;43:401-14.

32 Zhang L, Wu Y, Liu S, et al. Prevalence of depression in ankylosing spondylitis: a systematic review and meta-analysis. Psychiatry Investig 2019;16:565-74.

33 Sieper J, Rudwaleit M, Baraliakos X, et al. The assessment of spondyloarthritis International Society (ASAS) Handbook: a guide to assess spondyloarthritis. Ann Rheum Dis 2009;68:ii1-44.

34 Millner JR, Barron JS, Beinke KM, et al. Exercise for ankylosing spondylitis: an evidence-based consensus statement. Semin Arthritis Rheum 2016;45:411-27.

35 Karapolat H, Eyigor S, Zoghi M. Are swimming or aerobic exercise better than conventional exercise in ankylosing spondylitis patients? A randomized controlled study 2009;45:449-57. 